\title{
硫原子作为自由基受体在含硫杂环合成中的研究进展
}

\author{
杨文超*,a \\ 张明明 $a$ 陈 旺 $a$ 杨小虎 $b$ \\ 冯建国*, $a$ \\ $\left({ }^{a}\right.$ 扬州大学园艺与植物保护学院 江苏扬州 225009) \\ ( ${ }^{b}$ 浙江医院药剂科 杭州 310013)
}

\begin{abstract}
摘要 含硫杂环化合物如噻唑、噻吩和硫代黄酮等, 广泛存在于天然产物及生物活性分子中, 因其具有多样的生物活 性, 在药物化学、有机化学和功能性材料领域中的研究潜力越来越突出. 有效的含硫杂环合成策略已成为广泛研究的 主题. 不饱和烃的自由基串联反应构筑杂环衍生物一直是有机合成化学的一个重要研究分支, 近年来的研究成果也彰 显了其巨大的潜力. 研究者发现硫原子可以作为自由基的受体构建一个 $\mathrm{C}-\mathrm{S}$ 键，并通过该策略建立了一系列自由基串 联反应，其中基于茴香硫醚参与的不饱和烃自由基串联反应已逐渐发展成为一种重要的含硫杂环合成工具. 综述了该 领域的研究进展，根据茴香硫醚的成环类型，对近年来硫醚中硫原子作为自由基受体参与杂环合成的反应进行简要总 结, 并对反应设计、机理研究和研究展望等给予评述.
\end{abstract}

关键词 茴香硫醚; 杂环; 自由基串联反应; 双官能化

\section{Recent Progress in the Synthesis of Sulfur-Containing Heterocycles Using Sulfur Atom as Radical Acceptors}

\author{
Yang, Wenchao*,a Zhang, Mingming ${ }^{a} \quad$ Chen, Wang $^{a} \quad$ Yang, Xiaohu $^{b} \quad$ Feng, Jianguo $^{*, a}$ \\ ( ${ }^{a}$ School of Horticulture and Plant Protection, Yangzhou University, Yangzhou, Jiangsu 225009) \\ ( ${ }^{b}$ Department of Pharmacy, Zhejiang Hospital, Hangzhou 310013)
}

\begin{abstract}
Sulfur-containing heterocyclic compounds such as thiazole, thiophene, thiopyrone, widely exist in numerous natural products and biologically active molecules. Developing of effective strategies for the formation of sulfur-containing heterocycles has become a popular research hotspot. Meanwhile, the free-radical cascade reaction of unsaturated bonds in the synthesis of heterocycle has always been an important branch of synthetic chemistry, and the achieved progresses in recent years have also demonstrated its huge potential. Researchers found that sulfur atom could act as radical acceptors to build a $\mathrm{C}-\mathrm{S}$ bond, and established a series of free radical reactions through this strategy. Among them, the radical cascade reactions of anisole derivatives have been gradually developed into an important synthetic tool toward sulfur-containing heterocycles. The recent advances in the field of radical-initiated reactions of anisoles for the construction of sulfur-containing heterocycles, in which sulfur atoms play as radical acceptors, are summarized. In addition, the reaction design, mechanism and applicability of sulfur-containing heterocyclic compounds via radical cyclization towards the synthesis of complex molecules are also covered.
\end{abstract}

Keywords anisole; heterocycle; radical cascade reaction; difunctionalization

含硫杂环分子是重要的结构单元, 广泛存在于药 物、农用化学品、食品和材料分子中 ${ }^{[1]}$. 因此, 发展构建 含硫杂环的有效方法一直是工业界和学术界的关注焦 点之一 ${ }^{[2]}$. 近十年来, 自由基参与的含噻吩、噻唑环的构 筑与修饰已逐渐成为合成化学的研究热点, 并取得了一
系列研究进展, 涌现出诸多经典反应.

近年来，硫醚作为自由基前体和自由基受体的反应 已经得到诸多关注，其中以二芳基二硫醚参与的自由基 串联反应居多 ${ }^{[3]}$. 一般而言，二芳基二硫醚作为自由基 受体的化学反应是以原位产生的自由基加成到硫原子

\footnotetext{
* Corresponding authors. E-mail: wccyang@126.com; jgfeng@yzu.edu.cn Received May 16, 2020; revised June 12, 2020; published online July 22, 2020.

Project supported by the Natural Science Foundation of the Jiangsu Higher Education Institutions (No. 19KJB150020), the Natural Science Foundation for Young Scholars of Jiangsu Province (Nos. BK20170489, BK20190900) and the Agricultural Science and Technology Innovation Fund (No. CX(19)3112). 江苏省高校自然科学基金(No. 19KJB150020)、江苏省青年基金(Nos. BK20170489, BK20190900)和江苏省农业科技创新基金(No. CX(19)3112)资助项 目.
} 
上的形式进行, 这为含硫杂环的构建开辟了一条潜在的 新途径(Scheme 1). 王磊课题 组 ${ }^{[4]}$ 在 2016 报道了可见 光诱导醚的 $\alpha-\mathrm{C}\left(\mathrm{sp}^{3}\right)$ - $\mathrm{H}$ 硫醚化反应, 此反应是以硫原 子作为自由基受体的方式进行. 茴香硫醚(又名苯甲硫 醚) 作为硫醚的一种特殊骨架, 其用途广泛, 可作为新 一代抗生素罗非昔布的合成原料, 也是合成杀虫剂、杀 菌剂等的中间体, 其化学转换具有重要的研究价值 ${ }^{[5]}$. Zanardi 课题组 ${ }^{[6]}$ 早在 1985 年便发现邻甲硫基芳基重氮 盐可与炔烃发生自由基串联环化反应构筑苯并噻吩衍 生物, 在这个转化中, 自由基环化过程同样以自由基加 成到硫原子上同时释放甲基而终止. 与二芳基二硫醚相 比, 茴香硫醚中的硫原子作为自由基受体的研究较少, 研究结果的缺乏可能间接地误导研究者认为茴香硫醚 的活性和利用价值比二芳基二硫醚低. 这致使茴香硫醚 作为自由基受体合成含硫杂环在随后的近三十年里成 为一个近乎被遗忘的研究领域. 然而, 随着现代合成技 术的革新与广大化学家的关注, 硫原子作为自由基受体 的合成方法学和产物应用性被不断丰富, 这类方法构筑 硫杂环衍生物的重要性也在不断凸现. 目前, 该方法已 被成功应用于重要的硫杂环的构建, 例如苯并噻唑、苯 并噻吩和硫代黄酮(如 2-苯基-4H-硫代色烯-4-酮, 即黄 酮的环内氧原子被硫原子取代后的化合物)等其他含硫 杂环. 本文拟结合课题组的前期研究, 对茴香硫醚作为 自由基受体在硫杂环合成的研究进行综述. 根据自由基 串联反应产物类型, 主要分为两部分: 构建五元硫杂环 和构建六元硫杂环.

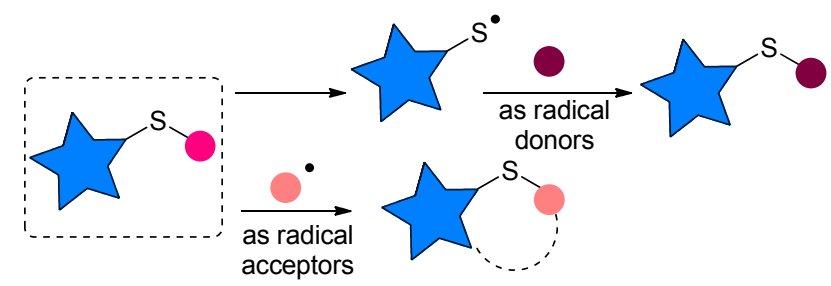

图式 1 硫醚参与的自由基反应

Scheme 1 Thioethers involved free radical reactions

\section{1 构建五元硫杂环}

\section{1 基于炔烃的自由串联构建苯并噻吩衍生物}

Zanardi 及其同事 ${ }^{[6]}$ 首次实现了铜粉或 $\mathrm{FeSO}_{4}$ 促进的 芳基重氮盐与炔烃的串联反应合成苯并噻吩衍生物, 作 者提出了硫原子作为自由基受体的可能反应机理, 为后 期研究打下了坚实的基础. 但该领域并未得到足够的重 视，在随后的二十多年时间里仅 McDonald 与 Schiesser 课题组 ${ }^{[7]}$ 分别独立探索了该反应, 并证实通过该策略亦 可获得相应的苯并硒吩衍生物(Scheme 2). 上述方法条 件比较苛刻，需使用大量的金属才能顺利完成反应，而
且底物的范围比较窄.

Zanardi, McDonald and Schiesser's work
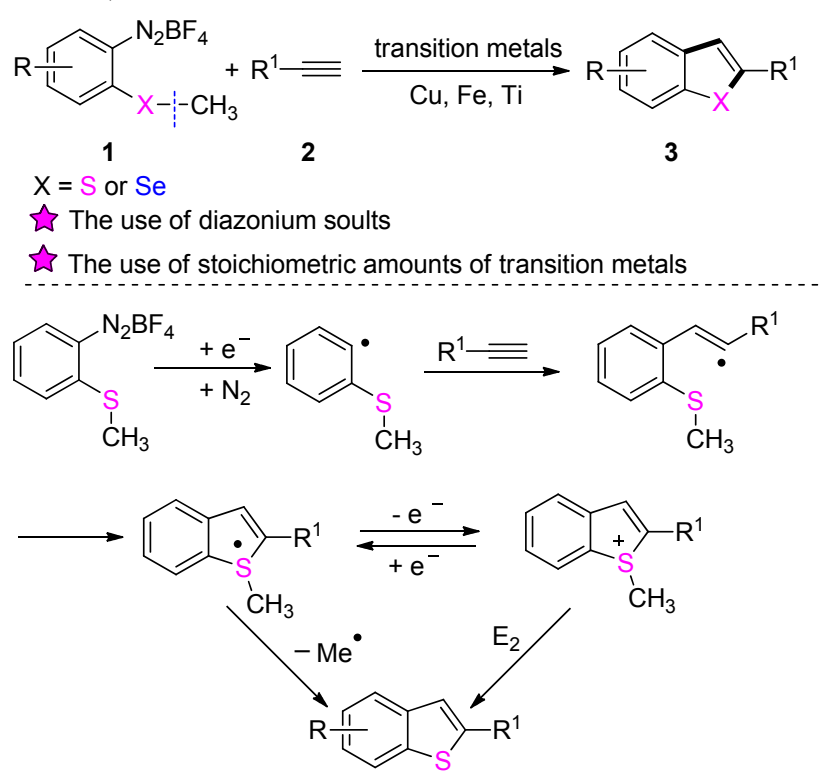

图式 2 邻甲基硫代芳基重氮盐与炔烃的自由基级联反应 Scheme 2 Radical cascade reaction between $o$-methylthioarene diazonium salts and alkyne

在过去十年中, 自由基串联反应引起了化学家的重 视，其中极具代表性的领域是光化学促进的自由基反 应，已逐渐发展成为一种重要的合成工具 ${ }^{[8-10]}$. 光化学 促进的硫原子作为自由基受体合成硫杂环的突破是 König 等 ${ }^{[11]}$ 于 2013 年发展的一种可见光诱导邻甲硫基 芳基重氮盐与炔烃的 $[3+2]$ 自由基环化反应(Scheme 3). 该反应条件温和，操作简便，使用廉价易得的有机染料 Eosin Y 作为催化剂, 以中等偏上的收率得到官能化苯
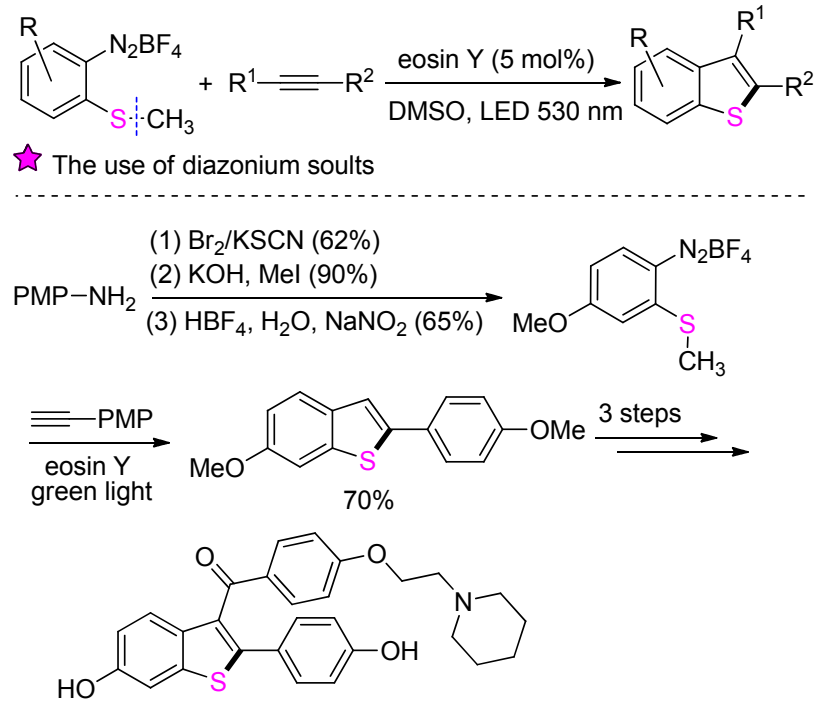

图式 3 光诱导的邻甲基硫代芳基重氮盐与炔烃的级联反应 Scheme 3 Visible-light initiated cascade reaction of $o$-methylthioarene diazonium salts and alkyne 
并噻吩衍生物. 此外, 该方法在雷洛昔芬药物关键中间 体的合成中也得到了应用，展现了较高的实用价值.

上述转换虽然在室温及无金属体系下进行，但是反 应需要预先制备芳基重氮盐, 增加了反应步骤, 其反应 成本相应增加. 2016 年, 张博课题组 ${ }^{[12]}$ 报道了有效且高 度实用的亚硝酸叔丁酯促进芳胺原位产生芳基自由基 参与炔烃的双官能化反应制备官能化苯并噻吩的方法 (Scheme 4), 相较于 König 等的工作, 该方法缩短了反 应步骤, 体现了步骤经济性.

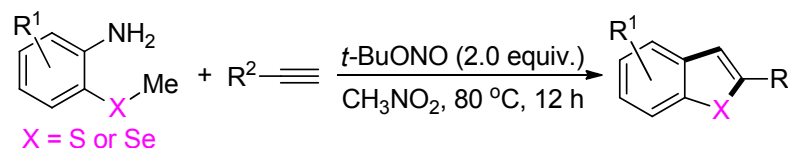<smiles>Fc1ccc2cc(-c3ccccc3)sc2c1</smiles>

$50 \%$ $45 \%$<smiles>FC(F)(F)c1ccc2oc(-c3ccsc3)cc2c1</smiles>
$43 \%$<smiles>CSc1cc2cc(C(F)(F)F)ccc2s1</smiles>

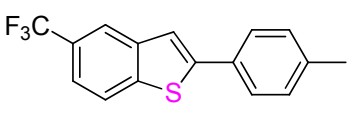

$68 \%$<smiles>c1ccc(-c2cc3ccccc3[se]2)cc1</smiles>

$50 \%$<smiles>CS(=O)(=O)c1cc2cc(C(F)(F)F)ccc2[se]1</smiles>

图式 4 苯胺与炔烃的自由基级联反应

Scheme 4 Radical cascade reaction between arylamines and alkynes

然而, 无论是预先制备的四氟化嗍重氮盐还是经由
芳胺原位生成的重氮盐, 在规模化生产时都有潜在的爆 炸危险，都需要小心操作，这在很大程度上限制了该方 法合成苯并噻吩的应用范围. 因此，亟需拓展新的反应 模式和新的反应试剂，通过硫原子淬灭自由基来合成硫 杂环. 另一方面, 由于砜基不仅存在于诸多天然产物中, 也是调节化合物生物活性的药效基团，在医药和农药领 域中有着重要的应用价值，对于砜基的引入已成为合成 化学的一个热点领域 ${ }^{[13]} .2017$ 年, 宋秋玲课题组 ${ }^{[14]}$ 设计 并合成了邻炔基茴香硫醚，并将其作为反应底物对该策 略的应用范围进行了拓展，成功建立了 3-砜基取代苯并 噻吩衍生物的合成新方法(Scheme 5, Condition A). 该反 应以乙腈为溶剂, 芳基亚磺酸在过氧化叔丁醇(TBHP) 促进下生成的砜基自由基参与炔烃的串联反应。同时该 反应底物展现出较好的官能团兼容性, 并以最高 $83 \%$ 的 产率得到各种骨架的苯并噻吩化合物. 此外，可以将该 策略从硫原子拓展到其同主族硒原子，成功合成 3-砜基 取代苯并硒吩衍生物.

虽然宋秋玲及其才队建立了这种高效的转换方法, 但反应中使用的过氧化物在高温下的潜在易爆性限制 了该反应的应用. 随后，该课题组 ${ }^{[15]}$ 在原有基础上继续 探索此类衍生物的合成与应用，利用苯磺酰氯取代亚磺 酸作为砜源, 在 $5 \mathrm{~W}$ blue LED 照射下产生的砜基自由 基对炔烃加成，并串联分子内环化获得 3-砜基取代苯并 噻吩衍生物(Scheme 5 Condition B). 首先, 激发态的光 敏催化剂 6 通过单电子转移被苯磺酰氯经过单电子转
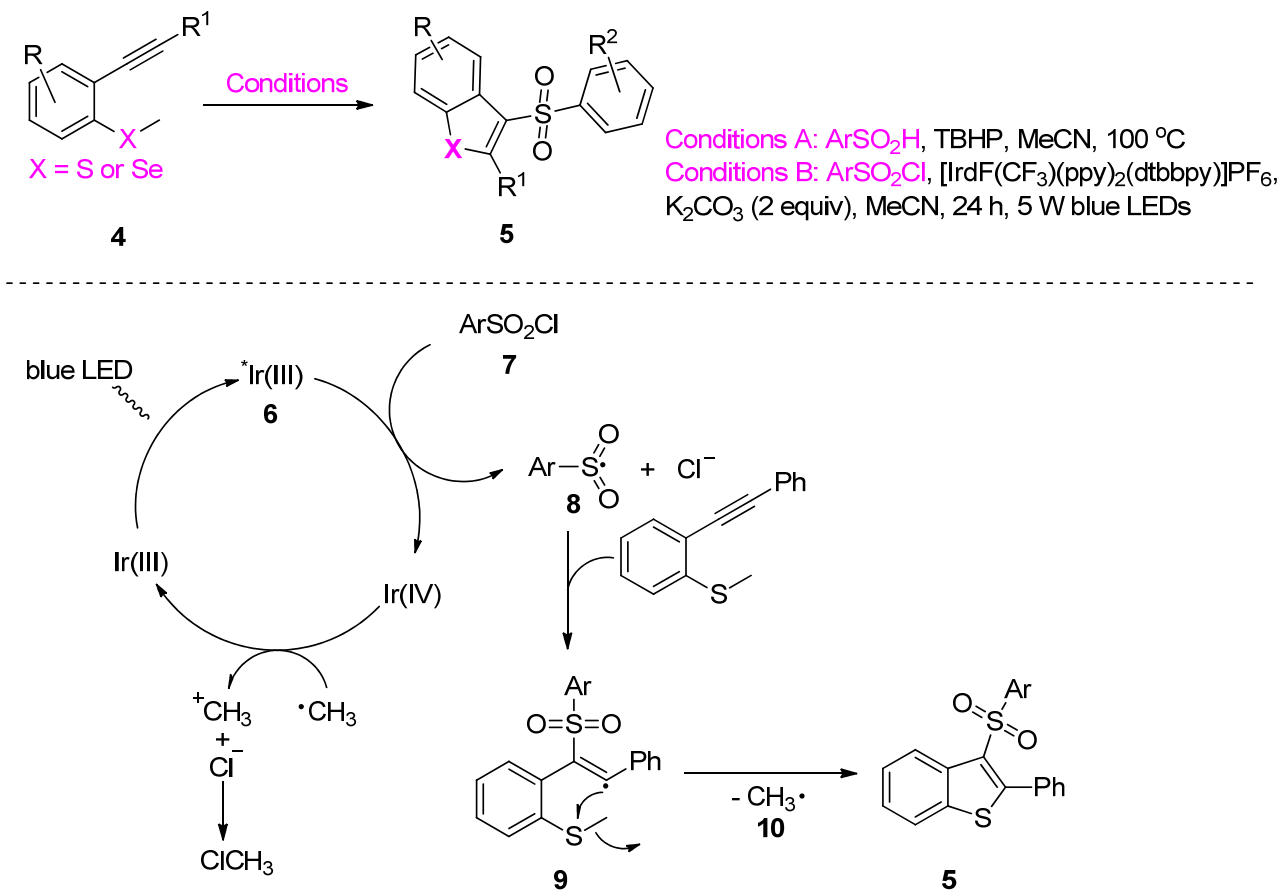

图式 5 由 2-炔基硫代苯甲醚合成 3-砜基苯并噻吩

Scheme 5 Synthesis of 3-sulfonyl benzothiophenes from 2-alkynyl thioanisoles 
移氧化后形成氧化态的光敏催化剂 $\operatorname{Ir}(\mathrm{IV})$, 同时产生的 砜基自由基 8 对底物的炔烃进行自由基插入, 得到自由 基中间体 9, 自由基中间体 9 被分子内的硫原子淬灭并 释放出甲基自由基 10. 离去的甲基自由基被氧化态的 光敏剂氧化为甲基正离子, 该物种最后与卤负离子形成 卤代物. 机理研究实验也捕捉并分离得到到砜基自由 基, 此外当硫原子的取代基团为 $\mathrm{Ph}$ 或 $\mathrm{Bn}$ 时, 反应体系 中可以检测到氯苯和芐溴的分子离子峰, 也间接证明了 机理的可能性.

吴华悦等 ${ }^{[16]}$ 实现了类似的转化, 反应以苯甲酰甲 酸 11 取代芳基亚磺酸钠和磺酰氯为底物, 在 $\mathrm{AgNO}_{3}$ 的 催化下, 以 $\mathrm{K}_{2} \mathrm{~S}_{2} \mathrm{O}_{8}$ 作为氧化剂, 氮气保护下加热反应至 $70{ }^{\circ} \mathrm{C}$, 最终以 $24 \% \sim 89 \%$ 的收率得到 3-酰基化的多取 代苯并噻吩衍生物 12 (Scheme 6, a). 在该体系下, 邻炔 基芳基硒醚与芳胺同样可以生成相应的苯并硒吩和吲 哚化合物. 作为茴香硫醚自由基串联环化反应的扩展, 随后该课题组发展了一种以硒粉作为硒源 (Scheme 6, b), 芳基硼酸在硝酸银催化下产生的芳基自由基 14 被 硒粉捕捉，得到芳基硒自由基 15, 该自由基随后参与 2炔基茴香硫醚的串联环化反应 ${ }^{[17]}$. 该方法底物范围广, 产率优越，操作简单，产物易于分离.
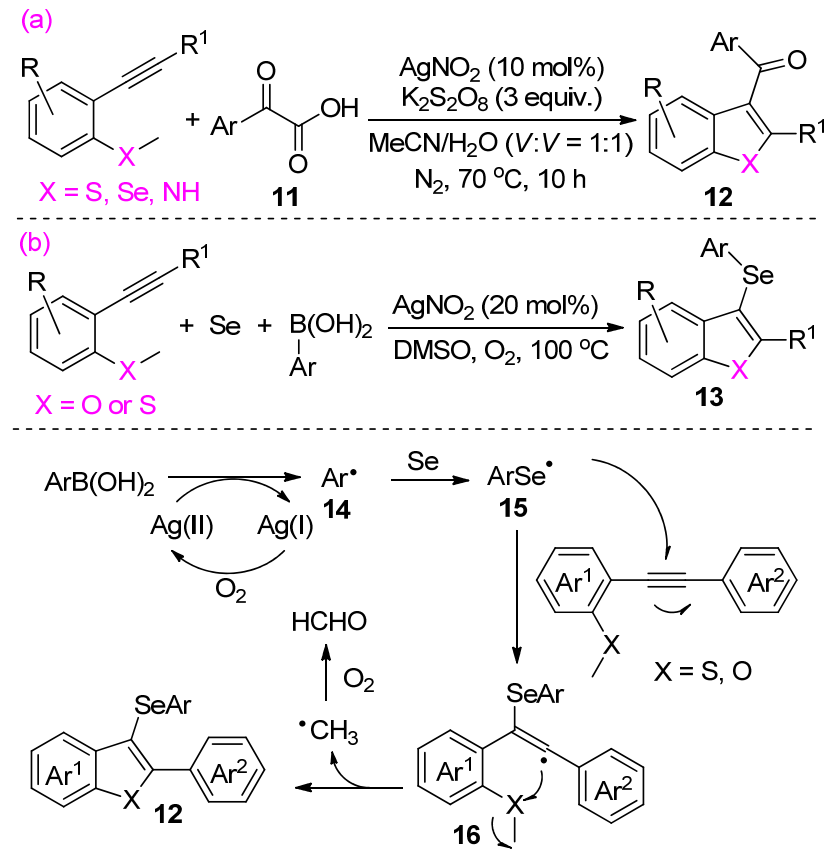

图式 6 由 2-炔基硫代苯甲醚合成取代苯并噻吩

Scheme 6 Synthesis of substituted benzothiophenes from 2alkynyl thioanisoles

近年来, 焦亚硫酸钠 $\left(\mathrm{Na}_{2} \mathrm{~S}_{2} \mathrm{O}_{5}\right)$ 作为一种存在广泛、 无毒且可再生的绿色无机二氧化硫源受到了合成化学 家的广泛关注 ${ }^{[18]}$. 在温和条件催化转化焦亚硫酸钠合 成高附加值的精细化学品, 成为绿色化学领域的前沿课
题. 2019 年, 吴劼课题组 ${ }^{[19]}$ 报道了可见光引发 $\mathrm{Ru}(\mathrm{bpy})_{3^{-}}$ $\mathrm{Cl}_{2}$ 催化 2-炔基茴香硫醚的自由基接力反应(Scheme 7), 在反应过程中，原位产生的甲基自由基 $\mathbf{1 0}$ 是关键中间 体，其与焦亚硫酸钠结合生成的甲砜基自由基 18 参与 2-炔基茴香硫醚的环化反应，并释放出另一甲基自由基 10 参与下一个接力循环. 虽然这种官能团转移反应展 现了较高的原子经济性，但是作者仅考察了硫原子上的 取代基为甲基和乙基的情况，且乙基取代时相应产物收 率偏低, 实际应用价值不大，因此探索其他官能团迁移 的耐受性尤为必要.

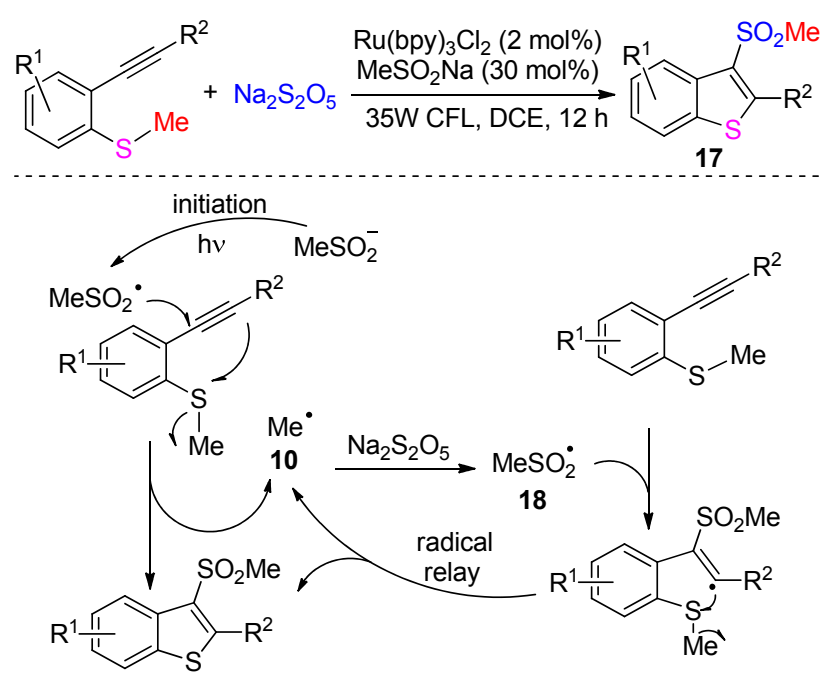

图式 72 2-炔基硫代苯甲醚的自由基串联反应 Scheme 7 Radical cascade reaction of 2-alkynyl thioanisoles

有机磷化合物被广泛应用于合成化学、材料、生物 医药、手性催化剂和农药等领域中 ${ }^{[20]}$. 简易高效地合成 有机磷化合物具有重要意义. 高玉珍及同事 ${ }^{[21]}$ 使用化 学剂量的氧化银使 2-炔基茴香硫醚发生磷酰基化/环化 反应 (Scheme 8), 以仲膦氧化物 19 作为磷酰基源合成了 3-磷酰基苯并噻吩 20. 仲膦氧化物是理想的磷酰化试 剂, 但为了激活它们的 $\mathrm{P}-\mathrm{H}$ 键, 往往需要化学剂量的 氧化剂. 这种自由基转换反应对磷酰基的稳定性敏感, 并不能将亚磷酸二乙酯类的底物引入苯并噻吩骨架. 此 外，该反应体系能较好地适用于苯并硒吩的合成，但无 法实现苯并呋喃的构筑.

\section{2 基于异腈的自由串联构建苯并噻唑衍生物}

异腈作为一氧化碳的等电子体, 是一个稳定的化合 物. 它具有离子型和卡宾型两种稳定的共振结构 (Scheme 9). 研究表明, 当异腈的结构以卡宾的形式存 在时，它是良好的自由基受体，生成亚胺自由基中间体， 继而发生后续串联反应 ${ }^{[22]}$. 在过去十年中, 异腈作为自 由基受体已被广泛应用于含氮杂环的构建, 例如吲哚、 喹诺酮、菲啶、喹喔啉、苯并咪唑等, 对于多聚杂原子 


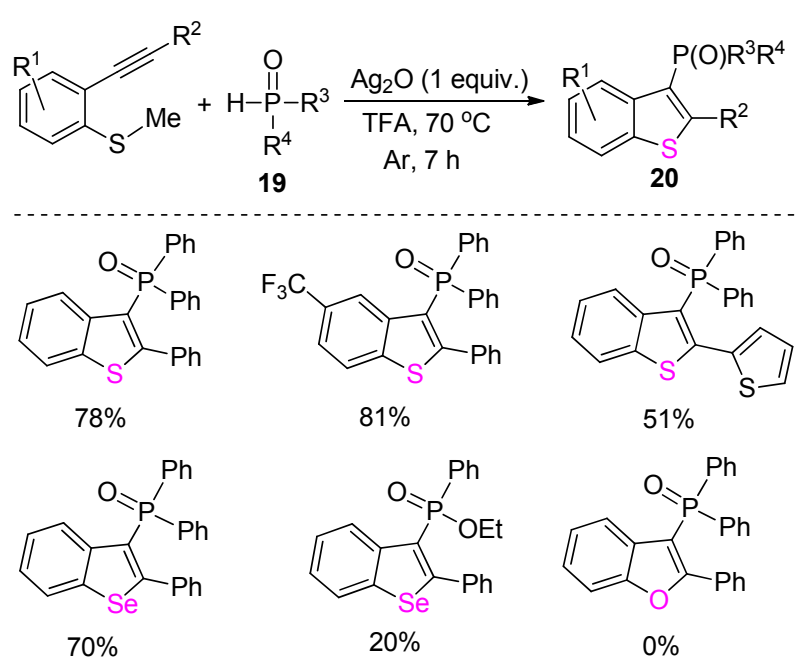

图式 8 2-炔基硫代苯甲醚的磷酰化反应

Scheme 8 Phosphorylation reaction of 2-alkynyl thioanisoles

环的构筑则未见报道. 如果由异腈形成的亚胺自由基加 成到邻位杂原子上，则可以构建一个碳-杂键，丰富了 官能化异腈的应用. 阻碍这一领域发展的原因可以归处 于以下两点: 裸露的杂原子(如氨基、羟基、颈基)和异 腈发生分子内的亲核取代反应产生卡宾中间体，阻碍了 自由基历程; 杂原子上的离去基团在亚胺自由基进攻时 必须容易离去且对最初产生的自由基物种呈现出惰性. 茴香硫醚的自由基淬灭性为发展异腈的自由基串联反 应构筑多聚杂原子环提供了无限可能.

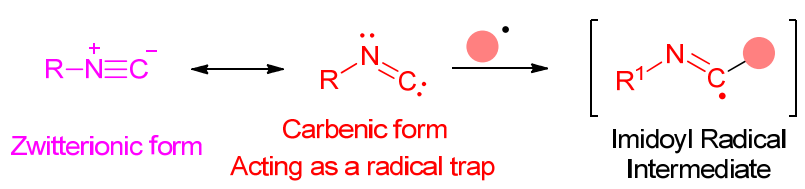

图式 9 异腈的存在形式

Scheme 9 Form of isocyanide

2018 年, 吴磊课题组 ${ }^{[23]}$ 首次在芳基异腈的邻位引 入甲硫基官能团用于捕捉异腈自由基插入形成的亚胺 自由基中间体 22, 在甲基自由基离去的同时构建一个 $\mathrm{C}-\mathrm{S}$ 键，合成了一系列 2-取代苯并噻唑衍生物 23 (Scheme 10). 该策略首次将异腈的自由基串联反应拓 展到多聚杂原子环合成. 发展这类方法学不仅可以极大 地丰富异腈的化学转化, 还可以构建一系列具有优势骨 架的杂环小分子化合物库, 为药物先导化合物的发现以 及进一步的开发提供可能. 在底物适用性方面, 多种自 由基源和取代的官能化异腈均能很好地转化得到相应 的串联目标产物. 为了验证反应机理作者做了系列控制 实验，作者首先向反应体系中加入自由基抑制剂四甲基 哌啶氧化物(TEMPO)或 2,6-二叔丁基-4-甲基苯酚(BHT) (Scheme 10, a), 无法获得相应的取代苯并噻唑产物. 此 外, 加入自由基捕捉剂 24, 通过 HRMS 可以检测到磷酰
基以及从硫醚上释放的甲基自由基物种 $25 \sim 28$ (Scheme 10, b). 鉴于此，作者推测了该反应可能的反应 机理. 在 $\mathrm{Mn}(\mathrm{OAc})_{3} \cdot 2 \mathrm{H}_{2} \mathrm{O}$ 促进下, 嗍酸或仲膦氧化物形 成芳基或磷酰基自由基中间体，之后与异腈发生自由基 插入得到亚胺自由基物种，该物种加成到硫醚的硫原子 上, 得到了最终目标产物并释放出甲基自由基.

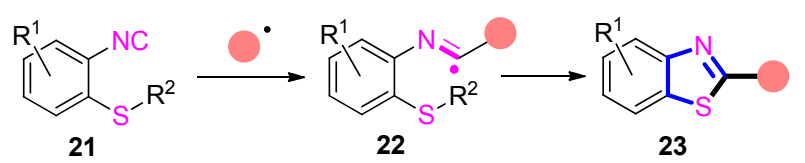

$\mathrm{R}^{2}=\mathrm{Me}, \mathrm{Et}, \mathrm{Bn}, \mathrm{Ph} \quad \mathrm{X}-\mathrm{O}=\mathrm{HP}(\mathrm{O}) \mathrm{Ar}_{2}$, aryl/alkyl boronic acid, etc.

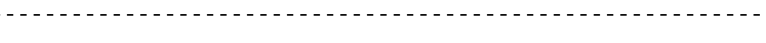

(a)

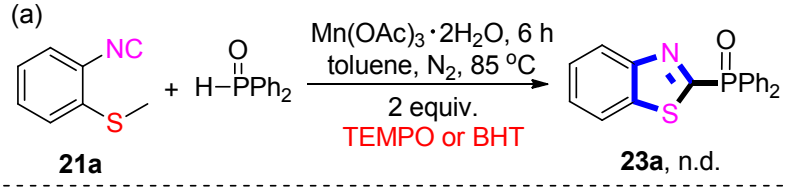

(b)
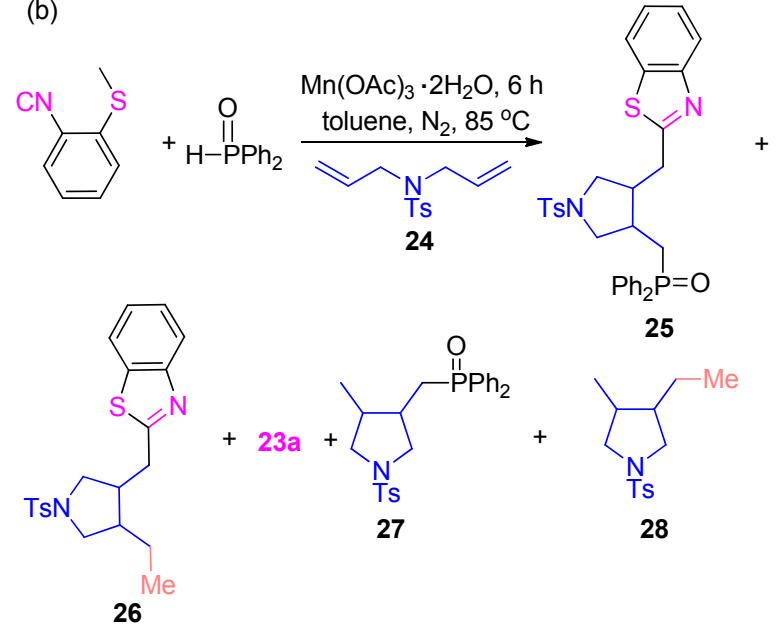

图式 10 2-异氰基芳硫醚的自由基环化合成 2-取代苯并噻唑 Scheme 10 Radical cyclization of 2-isocyanoaryl thioethers toward 2-substituted benzothiazoles

在开发以上新试剂的同时，科研工作者们也通过现 有的研究基础开发新的、便捷的反应模式合成同类产物, 比如将伯胺原位转化为异腈具有更高的步骤经济性，但 该类反应未见报道. 2018 年, 宋秋玲团队 ${ }^{[24]}$ 报道了 2-氨 基茴香硫醚 29 与溴二氟乙酸乙酯 30 的串联环化反应 (Scheme 11)，作者开创性地实现了溴二氟乙酸乙酯的 “双重身份”- - $\mathrm{C}(1)$ 合成子和二氟烷基化试剂，直接将 伯胺原位转换成异腈中间体, 继而与二氟烷基自由基串 联完成由芳胺直接到 2-取代苯并噻唑 31 的合成, 该反 应具有良好的官能团兼容性.

三氟甲基被认为是可以增强母体分子的代谢稳定 性、亲脂性和生物利用度的重要药效团. 此外，与全氟 烷基官能团的活性分子相比，二氟烷基官能团分子有着 截然不同的理化性质. 在氟原子的吸电子作用下，与氟 


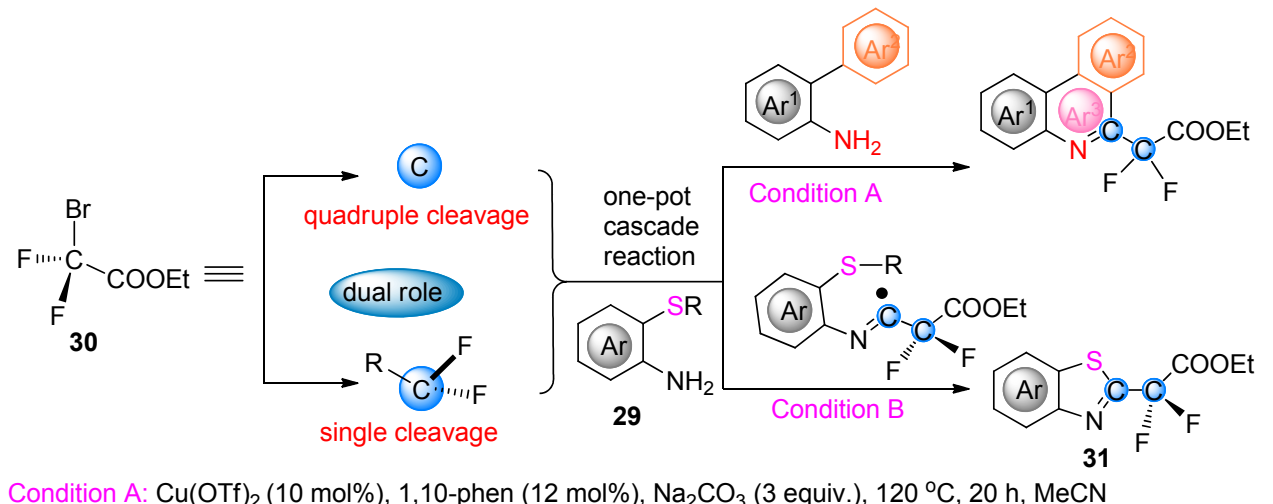

Condition B: $\mathrm{Cu}(\mathrm{OTf})_{2}(10 \mathrm{~mol} \%), 1,10-$ phen (12 mol\%), $\mathrm{Na}_{2} \mathrm{CO}_{3}$ (3 equiv.), $\mathrm{B}_{2} \mathrm{pin}_{2}(30 \mathrm{~mol} \%), 80^{\circ} \mathrm{C}, 20 \mathrm{~h}, \mathrm{MeCN}$

图式 11 串联法由芳胺与溴二氟乙酸乙酯获取有价值的产品

Scheme 11 Cascade approaches to access valuable products from arylamines with ethyl bromodifluoroacetate

相连的碳原子上的氢表现出一定的酸性, 易于形成分子 内或分子间氢键. 这些性质可能会提高含氟分子在生物 体内的代谢稳定性. 在吴否工作的启发下, 张兆国课题 组 ${ }^{[25]}$ 进一步探索了官能化异腈在含硫杂环合成中的应 用(Scheme 12). 在催化量的面式-三(2-苯基吡啶)合铱 配合物及 $7 \mathrm{~W}$ 蓝光照射下, 实现了 2-异氰基芳基硫醚的 自由基环化, 以较优越的收率合成了 $2-\mathrm{CF}_{3}$ 和 $2-\mathrm{CF}_{2} \mathrm{R}$ 取 代的苯并噻唑衍生物.
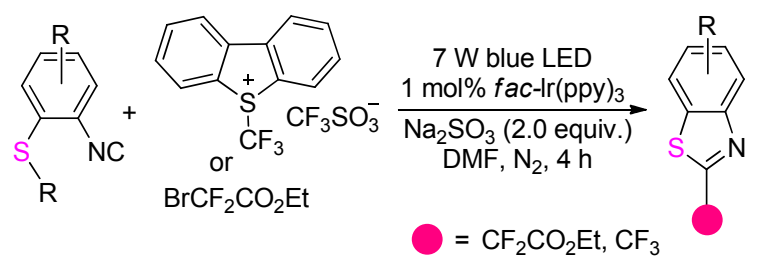

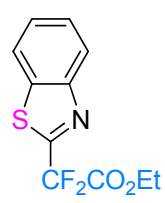

$93 \%$

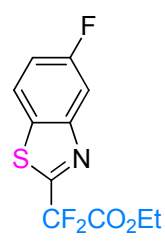

$67 \%$

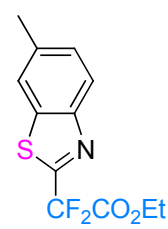

$80 \%$

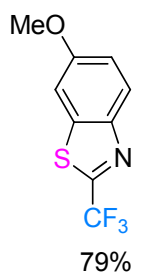

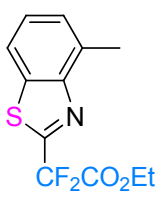

$82 \%$

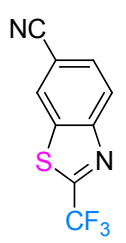

$77 \%$
图式 12 光诱导 2-异㲵基芳硫醚的自由基环化

Scheme 12 Visible-light initiated cascade reaction of 2-isocyanoaryl thioethers

虽然已经建立了光诱导的含氟苯并噻唑合成新方 法, 但是将该反应体系拓展至无催化剂体系以及全氟代 链状底物亦尤为必要. 2019 年, 陈晓岗与於兵课题组 ${ }^{[26]}$
发展了一例无金属和催化剂的 2-异氰基芳基硫醚与全 氟烷基碘代物的自由基加成/环化(Scheme 13), 合成了 一系列 2-氟烷基苯并噻唑. 该反应的特征在于有机碱 $N, N, N^{\prime}, N^{\prime}$-四甲基乙二胺(TMEDA)与底物 $\mathrm{R}_{\mathrm{f}}-\mathrm{I} 32$ 形成电 子给体-受体络合物 33, 该络合物经 $25 \mathrm{~W}$ 蓝光照射产生 相应的全氟烷基自由基 34. 作者提出了两种由 34 自由 基加成引发的串联路径. 在路径 A 中, 中间体 $\mathbf{3 7}$ 与 $\mathbf{3 5}$ 经历单电子转移形成 38 , 随后碘负离子进攻中间体 38 中的甲基碳得到目标产物与碘甲烷. 在路径 B 中，中间 体 37 与 35 通过协同质子偶合电子转移过程生成产物并 同时释放甲烷分子.

随后，杨文超及同事报道了另一个光引发的 2-异氰 基芳基硫醚与二芳基磷氧间的自由基串联反应 ${ }^{[27]}$, 反 应采用廉价的有机染料 Rose Bengal 作为光敏剂, 避免 了金属氧化剂的使用(Scheme 14), 产物中无金属残留, 从而更加适用于对金属残留量要求严格的药物合成领 域. 该研究中, 二芳基磷氧在光催化条件下产生磷酰基 自由基之后, 进一步与异腈发生自由基加成和分子内环 化/去甲基化, 产物的收率中等偏上, 底物适用范围广.

硫原子作为自由基受体构筑硫杂环被广泛地研究, 但在所有这些先前的研究中, 硫原子上的取代基团(甲 基、乙基、茮基和苯基)均以 “废弃物” 的形式从硫原 子上释放，对于硫原子上离去基团的再利用则很少报 道. 2019, 吴䂞课题组 ${ }^{[28]}$ 深入探索了 2-异氰基芳基硫醚 的自由基串联反应(Scheme 15), 实现了分子内官能团 转移, 在反应过程中内部自由基源( $\mathrm{R}$ 自由基)比源自过 氧化二叔丁基(DTBP)的甲基自由基具有更高的优先性. 与吴劼等 ${ }^{[19]}$ 的工作相比, 反应具有更高的官能团兼容 性, $\mathrm{R}$ 无论是诸如甲基、茮基、环己基的烷基还是芳基 均能高效地转换为相应产物. 


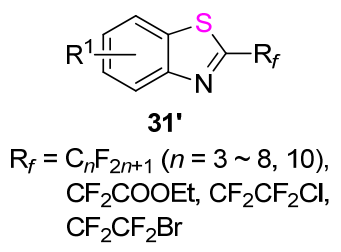

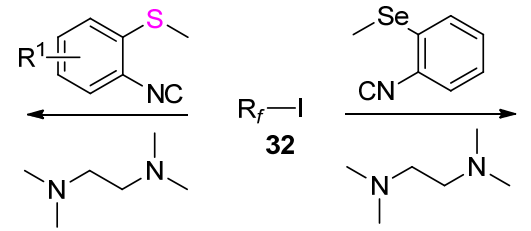

$25 \mathrm{~W}$ blue LEDs

$\mathrm{THF}, \mathrm{N}_{2}, 30^{\circ} \mathrm{C}, 3 \sim 12 \mathrm{~h}$

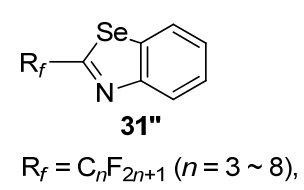

$\mathrm{R}_{f}=\mathrm{C}_{n} \mathrm{~F}_{2 n+1}(n=3 \sim 8)$,

$\mathrm{CF}_{2} \mathrm{COOEt}$<smiles>CP1[Te]=[Tl]1</smiles>

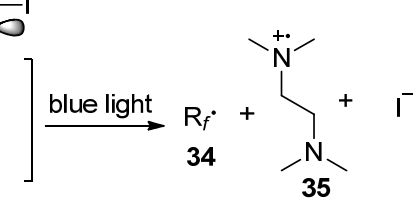

EDA complex<smiles>[R]C1=Nc2ccccc2[Si]1(C)CC[Si]C(C)N(C)CC(C)C(C)C(C)C</smiles>

37<smiles></smiles><smiles></smiles>

38

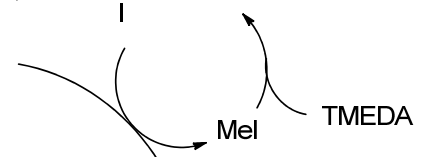

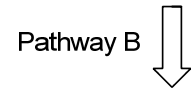

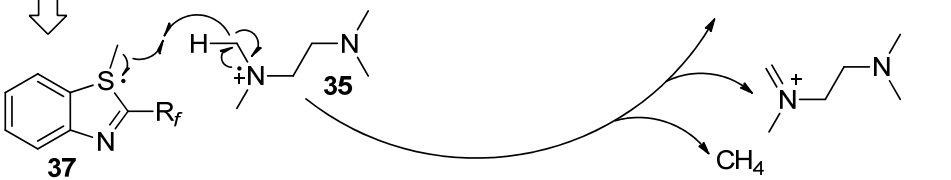

图式 13 光诱导 2-异氰基芳硫醚的自由基环化

Scheme 13 Visible-light initiated cascade reaction of 2-isocyanoaryl thioethers

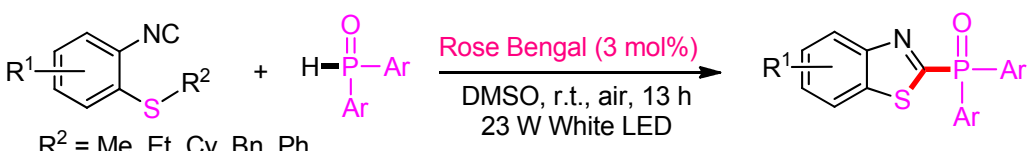

$\mathrm{R}^{2}=\mathrm{Me}, \mathrm{Et}, \mathrm{Cy}, \mathrm{Bn}, \mathrm{Ph}$

图式 14 2-异氰基芳硫醚的磷酰化反应

Scheme 14 Phosphorylation reaction of 2-isocyanoaryl thioethers
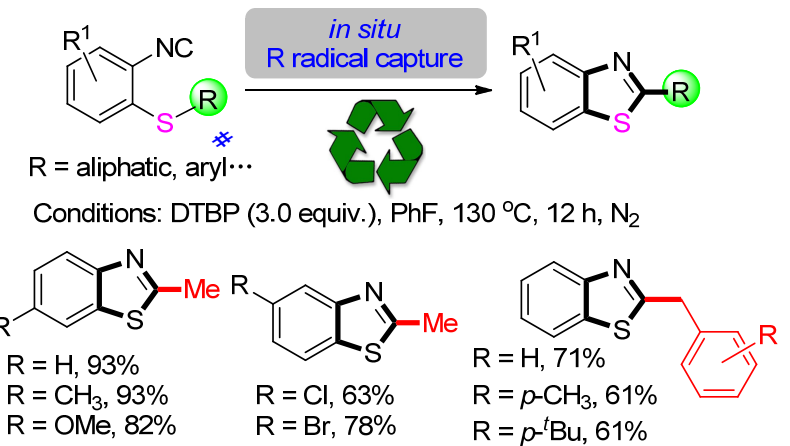

图式 152 -异氧基芳硫醚的官能团转移环化反应 Scheme 15 Group-transfer cyclization of 2-isocyanoaryl thioethers

\section{3 基于 1,7-烯炔的自由串联构建噻吩并喹啉酮衍生 物}

这些高效含硫杂环的构建方法充分体现了硫原子 作为自由基受体反应的优越性，也吸引了化学家们对于 底物多样性的设计与探索. 2019 年, 李金恒课题组 ${ }^{[29]}$ 报 道了一种新颖的 1,7-烯炔的无金属氧化 $[2+2+1]$ 杂环 化反应合成硫杂环的方法(Scheme 16), 反应使用过氧 化苯甲酰(BPO)作为氧化剂, 以硫氰酸钠作为硫源, 在 单一反应中形成三个化学键(两个 $\mathrm{C}-\mathrm{S}$ 键与一个 $\mathrm{C}-\mathrm{C}$ 键). 反应通过烯烃和芳基之间的轨道相互作用加速环 化, 有利于硫氰基化反应以及分子内 $\mathrm{C}-\mathrm{S}$ 键形成. 可 
能的机理如 Scheme 16 所示. 首先, BPO 受热均裂为苯 甲酰氧基自由基, 其氧化 $\mathrm{NaSCN}$ 为 $\mathrm{SCN}$ 自由基 41. 然 后, 41 与 1,7-烯炔 39 发生分子间加成产生自由基中间体 42, 中间体 42 经历分子内自由基环化得到烯基自由基 中间体 43, 随后 43 分子内加成到硫原子上得到硫杂环 中间体 44. 最后 44 被 BPO 氧化为正离子并脱去氧基得 到目标产物 40.

如上文所述，虽然市售的二芳基二硫醚作为自由基 受体构建 $\mathrm{C}-\mathrm{S}$ 键已被建立且常见于文献报道, 但是通 过裂解 $\mathrm{S}-\mathrm{S}$ 键和 $\mathrm{C}-\mathrm{S}$ 键作为硫源来合成含硫杂环的 例子却很少见. 李金恒等 ${ }^{[30]}$ 在他们前期的研究基础上, 探索了以二芳基二硫醚作为硫源, 在过氧化苯甲酸叔丁 酯(TBPB) 促进下实现 1,7-烯炔的 $[2+2+1]$ 自由基杂
环化反应(Scheme 17). 该课题组利用这一策略实现了 不同取代噻吩并喹啉酮衍生物的合成，表明这类方法对 于二芳基二硫醚类化合物有非常好的兼容性.

\section{2 构建六元硫杂环}

从合成化学的角度来说, 炔烃自由基的串联反应一 直占据重要的地位，其可以非常方便、高效地用于构建 多样的含螺(杂)环结构中间体，继而发生后续的化学转 化，完成从相对廉价的炔烃到高附加值、结构复杂分子 的高效转化，为具有生物活性或者药物活性的分子提供 重要的合成方法 ${ }^{[31]}$. 硫原子作为自由基受体构建硫杂 环的发展不仅局限在建立绿色、温和、高效的反应模式, 更在于反应底物的设计，从而丰富硫杂环种类. 2018 年，<smiles>[R]C#Cc1ccccc1N([R])C(=O)C([R])=C</smiles>

39<smiles>[R]C1=C2c3cccc([R])c3N([R])C(=O)C2([R])CSC1[R19]</smiles>

40

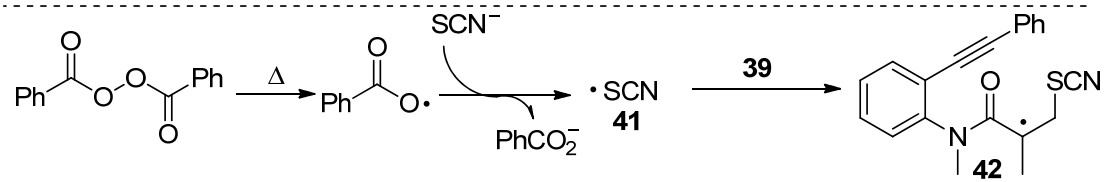<smiles>CC(C)[C@H](Cc1ccccc1)OC(=O)[O-]</smiles><smiles>CN1C(=O)C(C)(C)C(=C(c2ccccc2)S(C)(=O)=O)c2ccccc21</smiles>

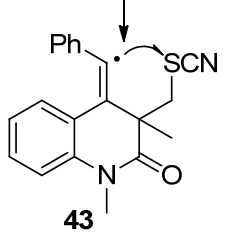

图式 16 1,7-烯炔与硫氰酸盐的 $[2+2+1]$ 杂环化

Scheme $16[2+2+1]$ Heteroannulation of 1,7-enynes with thiocyanates<smiles>[R]C#Cc1ccccc1N([R])C(=O)C([R])C(=C)[SH+]Sc1ccccc1</smiles><smiles>[R]CC1([R])C(=O)N([R])c2ccccc2C1=C1[R]#CCC1</smiles><smiles>CN1C(=O)C2(C)CSC(c3ccccc3)=C2c2ccccc21</smiles>

$90 \%$

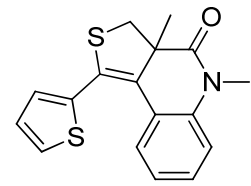

$68 \%$<smiles>Cc1ccccc1C1=C2c3ccccc3N(C)C(=O)C2(C)CS1</smiles>

$92 \%$<smiles>COc1cccc(C2=C3c4ccccc4N(C)C(=O)[C@@]3(C)CS2)c1</smiles>

$71 \%$<smiles>CN1C(=O)C2(C)CSC(c3ccccn3)=C2c2ccccc21</smiles>

$60 \%$<smiles>CN1C(=O)C2(C)CSC(c3ccsc3)=C2c2ccccc21</smiles>

$78 \%$<smiles>CCCCCCC1=C2c3ccccc3N(C)C(=O)C2(C)CS1</smiles>

$69 \%$<smiles>CCCCCCCC1=C2c3ccccc3N(C)C(=O)C2(C)CS1</smiles>

图式 17 1,7-烯炔与二芳基二硫醚的 $[2+2+1]$ 杂环化

Scheme $17[2+2+1]$ Heteroannulation of 1,7-enynes with disulfanes 
高玉珍和李纲课题组 ${ }^{[32]}$ 报道了苯乙炔基硫烷 46 与不饱 和 $\alpha$-溴羰基化合物 47 的分子间串联双环化反应，构筑 了硫代苯并双环骨架 48 (Scheme 18), 首次将该系列策 略从五元硫杂环的构建拓展至六元硫杂环的构建. 作者 通过详细的机理研究, 阐述了生成目标产物所经历的可 能路径. 首先, 底物 47 与 $\mathrm{Cu}(\mathrm{I})$ 催化剂之间通过单电子 转移生成相应的烷基自由基, 随后烷基自由基 49 加成 到 46 中的炔烃并继而发生分子内的环化得到末端烷基
自由基 51. 然后，该自由基物种被硫原子捕捉，形成六 元硫杂环自由基中间体 52, 该物种随后经历 $\mathrm{Cu}(\mathrm{II})$ 氧化 与脱甲基化生成目标产物.

2019 , 宋秋玲等 ${ }^{[33]}$ 在其前期对硫原子作为自由基 受体构建杂环的研究基础上，进一步设计反应底物，探 索了把硫原子作为自由基受体应用到炔酮衍生物的串 联反应中，成功合成了硫代黄酮化合物(Scheme 19，a). 这一合成方法具有优越的底物普适性，一系列自由基前

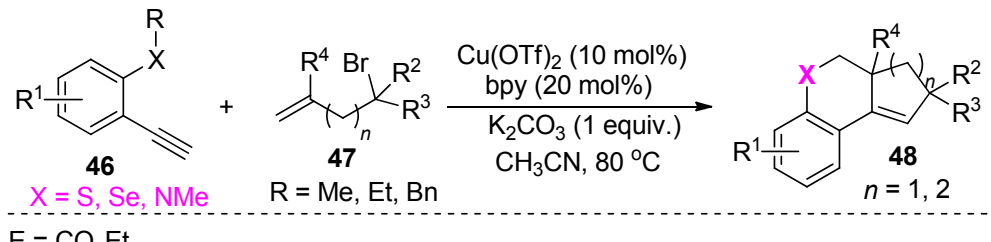

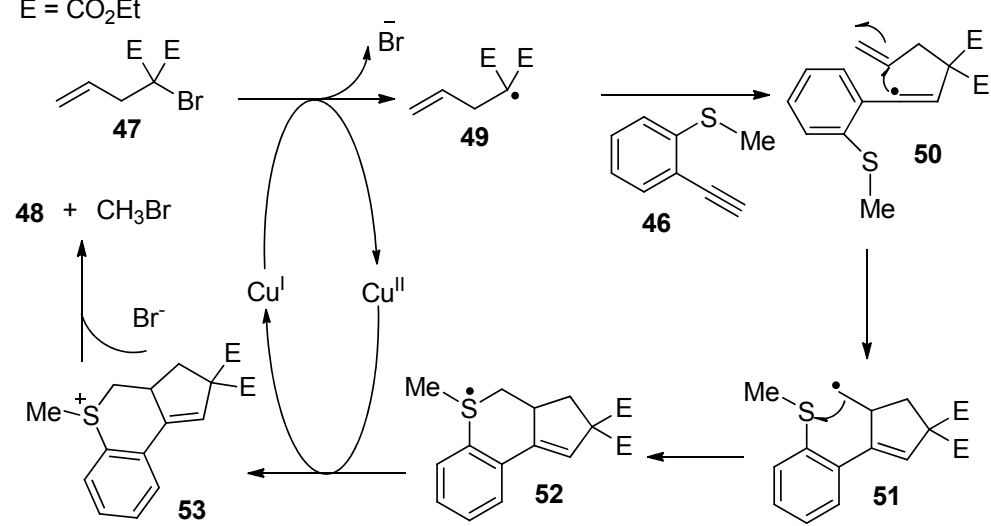

图式 18 通过硫醚参与的自由基反应合成化合物 $\mathbf{4 8}$

Scheme 18 Synthesis of compound $\mathbf{4 8}$ by free radical reaction involving thioethers

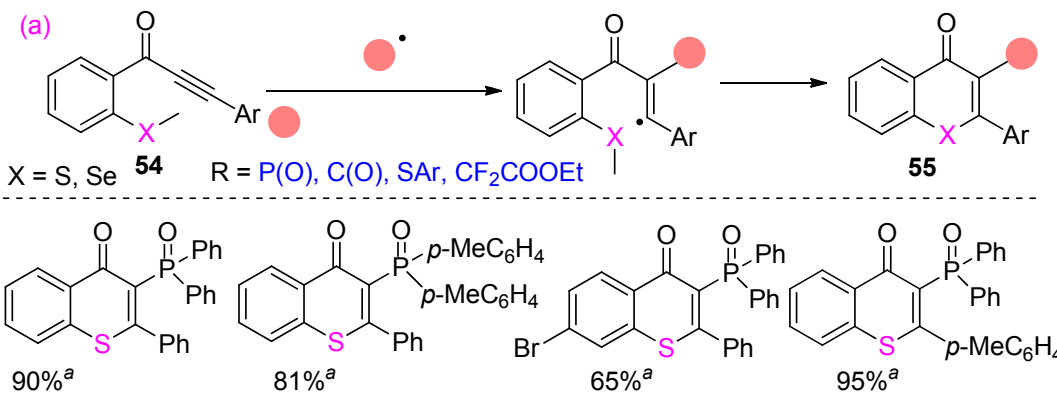<smiles>O=c1c(-c2ccccc2)c(-c2ccccc2)sc2ccccc12</smiles>

$88 \%^{b}$<smiles>O=C(c1ccccc1)c1c(-c2ccccc2)sc2ccccc2c1=O</smiles>

$62 \%^{\mathrm{C}}$<smiles>CCOc1sc2ccccc2c(=O)c1C(F)(F)F</smiles>

$70 \%^{d}$<smiles>N#CCc1c(-c2ccccc2)sc2ccccc2c1=O</smiles>

$92 \%{ }^{\mathrm{e}}$

a: $\mathrm{R}_{2} \mathrm{P}(\mathrm{O}) \mathrm{H}, \mathrm{Ag}_{2} \mathrm{CO}_{3}(10 \mathrm{~mol} \%), \mathrm{Zn}\left(\mathrm{NO}_{3}\right)_{2} \cdot \mathrm{H}_{2} \mathrm{O}(10 \mathrm{~mol} \%), \mathrm{MeCN}, 100^{\circ} \mathrm{C}, 12 \mathrm{~h}, \mathrm{Ar}$;

${ }^{b}$ : ArSH, TBHP (1.5 equiv.), $\mathrm{CH}_{3} \mathrm{CN}(2 \mathrm{~mL}), 80^{\circ} \mathrm{C}, 12 \mathrm{~h}, \mathrm{~N}_{2}$;

c: $\operatorname{ArCHO}, \operatorname{TBAB}(0.2 \mathrm{mmol}), \mathrm{K}_{2} \mathrm{~S}_{2} \mathrm{O}_{8}$ (2 equiv.), DCE $(2 \mathrm{~mL}), 90^{\circ} \mathrm{C}, 12 \mathrm{~h}, \mathrm{~N}_{2}$;

d: $\mathrm{BrCF}_{2} \mathrm{CO}_{2} \mathrm{Et}, \mathrm{Ir}(\mathrm{ppy})_{3}(2 \mathrm{~mol} \%), \mathrm{K}_{2} \mathrm{HPO}_{4}$ (2 equiv.), DMF ( $2 \mathrm{~mL}$ ), r.t., $12 \mathrm{~h}, 10 \mathrm{~W}$ blue LED, $\mathrm{N}_{2}$;

e: $\mathrm{CH}_{3} \mathrm{CN}(2 \mathrm{~mL})$, TBPB (2 equiv.), $130^{\circ} \mathrm{C}, \mathrm{Ar}, 12 \mathrm{~h}$.

(b) $54+\mathrm{AgSCF}_{3} \underset{\mathrm{CH}_{3} \mathrm{CN}, 80^{\circ} \mathrm{C}, 12 \mathrm{~h}, \mathrm{~N}_{2}}{\mathrm{NH}_{2} \mathrm{~S}_{2} \mathrm{O}_{8}(1.5 \text { equiv. }} \mathrm{55}: \mathrm{-SCF}$

图式 19 通过硫醚参与的自由基反应合成化合物 $\mathbf{5 5}$

Scheme 19 Synthesis of compound 55 by free radical reaction involving thioethers 
体如乙腈、溴二氟乙酸乙酯、二苯基磷氧、硫酚和醛等 都可以在该反应体系中实现转化. 除此之外, 反应也展 现出较好的官能团兼容性, 无论是与炔酮羰基直接相连 的芳环还是与炔烃相连的芳环上接有不同取代基时, 都 可以获得 $60 \% \sim 95 \%$ 的收率. 最近, 黄焰根课题组 ${ }^{[34]}$ 成 功将该自由基串联方法拓展至 3-三氟甲硫基硫代黄酮 与苯并噻吩化合物的合成中(Scheme 19, b).

最近, 於兵及其同事 ${ }^{[35]}$ 继续探索此类衍生物的绿 色、高效合成与应用, 报道了含砜与含磷硫代黄酮的光 催化合成(Scheme 20). 该系列策略使用绿色环保的水 或乙二醇作为反应溶剂, 解决了传统方法需要使用有机 溶剂的局限, 为磷酰基、砜基自由基参与的不饱和烃串 联环化反应提供了有效参考. 值得一提的是反应甚至可 以放大量合成至 $5 \mathrm{mmol}$, 并以 $67 \%$ 的收率得到相应的 含磷产物 $1.46 \mathrm{~g}$.

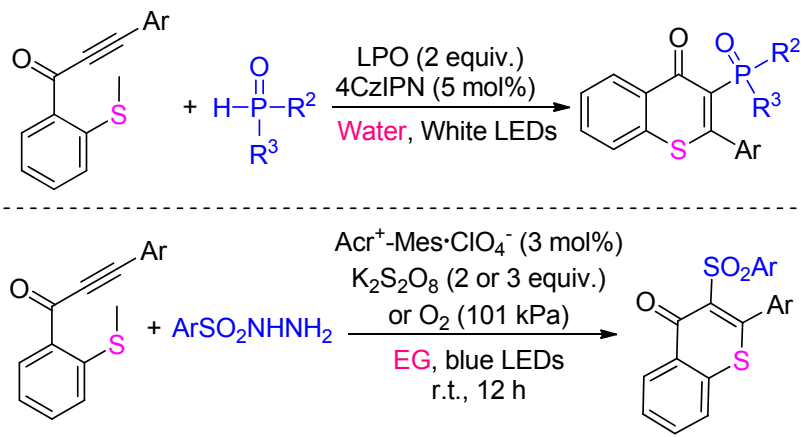

图式 20 无金属体系合成 2-取代硫代黄酮

Scheme 20 Metal-free synthesis of 2-substituted thioflavones

\section{3 总结与展望}

硫原子作为自由基受体用于制备硫杂环从 20 世纪 90 年代开始就已经有人在研究, 但真正引起合成化学 家的重视发生在近十年, 一些实用且高效的硫杂环合成 策略相继被报道. 然而, 这些方法至今尚未足够完善以 达到工业生产的需求, 存在着或多或少的缺点, 例如依 赖金属催化剂、催化体系复杂和反应条件苛刻等. 随着 合成化学的发展及新反应体系(光催化化学、电催化化 学、光电化学)逐渐地被建立, 相信会有更多的研究成果 被报道出来并攻克相应的难题. 此外, 虽然硫原子作为 自由基受体已被证实在硫杂环合成中具有较高的优越 性, 但受限于反应底物的设计, 产物分子结构的局限性 也比较大, 因此还有待合成化学家们设计新的反应底 物, 发展新的反应模式、新的催化体系来突破这些瓶颈 构筑结构更为新颖、复杂的硫杂环化合物. 值得指出的 是, 通过此策略构建具有手性季碳中心的含硫杂环至今 尚未取得实质性的进展，仍有很大的提升空间.

\section{References}

[1] (a) Mishra, R.; Sachan, Neetu.; Kumar, N.; Mishra, I.; Chand, P. J. Heterocycl. Chem. 2018, 55, 2019.

(b) Zhao, F.; Hu, Y.; Li, Q.; Hu, S. Chin. J. Org. Chem. 2020, 40, 1068 (in Chinese).

(赵芳, 胡洋, 李巧, 胡胜利, 有机化学, 2020, 40, 1068.)

(c) He, W.; Liu, D.; Gan, X.; Zhang, J.; Liu, Z.; Yi, C.; Song, B. Chin. J. Org. Chem. 2019, 39, 2287 (in Chinese)

(何文静, 刘登曰，甘秀海，张建，刘峥军，易崇粉，宋宝安，有 机化学, 2019, 39, 2287.)

(d) Feng, M.; Tang, B.; Liang, S. H.; Jiang, X. Curr. Top. Med. Chem. 2016, 16, 1200

(e) Liu, H.; Gao, Y.; Cao, J.; Li, T.; Wen, Y.; Ge, Y.; Zhang, L.; Pan, G.; Zhou, T.; Yang, B. Mater. Chem. Front. 2018, 2, 1853.

[2] (a) Chauhan, P.; Mahajan, S.; Enders, D. Chem. Rev. 2014, 114, 8807.

(b) Liu, H.; Jiang, X. Chem.-Asian J. 2013, 8, 2546.

[3] (a) Xiao, Z.; Wang, L.; Wei, J.; Ran, C.; Liang, S.; Shang, J.; Chen, G.-Y.; Zheng, C. Chem. Commun. 2020, 56, 4164.

(b) Vara, B. A.; Li, X.; Berritt, S.; Walters, C. R.; Petersson, E. J.; Molander, G. A. Chem. Sci. 2018, 9, 336

[4] Zhu, X.; Xie, X.; Li, P.; Guo, J.; Wang, L. Org. Lett. 2016, 18 1546.

[5] Zan, N.; Xie, D.; Li, M.; Jiang, D.; Song, B. J. Agric. Food Chem. 2020, 68, 6280 .

[6] Leardini, R.; Pedulli, G. F.; Tundo, A.; Zanardi, G. J. Chem. Soc., Chem. Commun. 1985, 1390.

[7] (a) McDonald, F. E.; Burova, S. A.; Huffman, L. G. Jr. Synthesis 2000, 970.

(b) Staples, M. K.; Grange, R. L.; Angus, J. A.; Ziogas, J.; Tan, N. P. H.; Taylor, M. K.; Schiesser, C. H. Org. Biomol. Chem. 2011, 9, 473.

[8] (a) Chen, Y.; Lu, L.-Q.; Yu, D.-G.; Zhu, C.-J.; Xiao, W.-J. Sci. China: Chem. 2019, 62, 24.

(b) Shang, T.; Lu, L.; Cao, Z.; Liu, Y.; He, W.; Yu, B. Chem. Commun. 2019, 55, 5408 .

(c) Xu, C.-H.; Li, Y.; Li, J.-H.; Xiang, J.-N.; Deng, W. Sci. China: Chem. 2019, 62, 1463 .

(d) Yang, W.-C.; Feng, J.-G.; Wu, L.; Zhang, Y.-Q. Adv. Synth. Catal. 2019, 361, 1700 .

(e) Li, D.; Yang, W.-C. Tetrahedron Lett. 2019, 60, 1792.

(f) Zhang, Y.; Sun, K.; Lv, Q.; Chen, X.; Qu, L.; Yu, B. Chin. Chem. Lett. 2019, 30, 1361.

(g) Ruan, L.; Liu, C.; Sun, J.; Zhou, M. Chin. J. Org. Chem. 2019, 39, 2403 (in Chinese).

(阮利衡, 刘畅, 孙京, 周明东, 有机化学, 2019, 39, 2403.)

(h) Xiong, L.; Hu, H.; Wei, C.-W.; Yu, B. Eur. J. Org. Chem. 2020, 1588 .

[9] Luo, K.; Yang, W.-C.; Wu, L. Asian J. Org. Chem. 2017, 6, 350.

[10] (a) Chen, J.-R.; Hu, X.-Q.; Lu, L.-Q.; Xiao, W.-J. Acc. Chem. Res. 2016, 49, 1911.

(b) Wu, Y.; Chen, J.-Y.; Li, Q.; Wei, W.-T. Chin. J. Org. Chem. 2020, 40, 589 (in Chinese).

(吴燕, 陈锦杨, 李强, 魏文廷, 有机化学, 2020, 40, 589.)

[11] Hari, D. P.; Hering, T.; König, B. Org. Lett. 2012, 14, 5334.

[12] Zang, H.; Sun, J.-G.; Dong, X.; Li, P.; Zhang, B. Adv. Synth. Catal. 2016, 358,1746

[13] (a) Zhu, J.; Yang, W.-C.; Wang, X.-D.; Wu, L. Adv. Synth. Catal. 2018, 360, 386.

(b) Ye, S.; Qiu, G.; Wu, J. Chem. Commun. 2019, 55, 1013.

(c) Yang, W.-C.; Dai, P.; Luo, K.; Wu, L. Adv. Synth. Catal. 2016, $358,3184$.

(d) Qiu, G.; Zhou, K.; Wu, J. Chem. Commun. 2018, 54, 12561.

[14] Xu, J.; Yu, X.; Yan, J.; Song, Q. Org. Lett. 2017, 19, 6292.

[15] Yan, J.; Xu, J.; Zhou, Y.; Chen, J.; Song, Q. Org. Chem. Front. 2018, 5, 1483.

[16] Liu, W.; Hu, Y.-Q.; Hong, X.-Y.; Li, G.-X.; Huang, X.-B.; Gao, 
W.-X.; Liu, M.-C.; Xia, Y.-Z.; Zhou, Y.-B.; Wu, H.-Y. Chem. Commun. 2018, 54, 14148.

[17] An, C.; Li, C.-Y.; Huang, X.-B.; Gao, W.-X.; Zhou, Y.-B.; Liu, M.-C.; Wu, H.-Y. Org. Lett. 2019, 21, 6710.

[18] Ye, S.; Yang, M.; Wu, J. Chem. Commun. 2020, 56, 4145.

[19] Gong, X.; Wang, M.; Ye, S.; Wu, J. Org. Lett. 2019, 21, 1156.

[20] Gao, Y.; Tang, G.; Zhao, Y. Chin. J. Org. Chem. 2018, 38, 62 (in Chinese).

(高玉珍，唐果，赵玉芬，有机化学，2018，38，62.)

[21] Cai, T.; Liu, J.; Zhang, H.; Wang, X.; Feng, J.; Shen, R.; Gao, Y. Org. Lett. 2019, 21, 4605.

[22] (a) Song, B.; Xu, B. Chem. Soc. Rev. 2017, 46, 1103.

(b) Zhang, B.; Studer, A. Chem. Soc. Rev. 2015, 44, 3505.

(c) Lei, J.; Huang, J.; Zhu, Q. Org. Biomol. Chem. 2016, 14, 2593.

(d) Li, D.; Mao, T.; Huang, J.; Zhu, Q. Org. Lett. 2017, 19, 3223.

(e) Li, Y.; Miao, T.; Li, P.; Wang, L. Org. Lett. 2018, 20, 1735.

[23] Yang, W.-C.; Wei, K.; Sun, X.; Zhu, J.; Wu, L. Org. Lett. 2018, 20, 3144.

[24] Ma, X.; Mai, S.; Zhou, Y.; Cheng, G.-J.; Song, Q. Chem. Commun. 2018, 54, 8960 .

[25] Yuan, Y.; Dong, W.; Gao, X.; Xie, X.; Zhang, Z. Org. Lett. 2019, 21,469 .

[26] Liu, Y.; Chen, X.-L.; Sun, K.; Li, X.-Y.; Zeng, F.-L.; Liu, X.-C.; Qu, L.-B.; Zhao, Y.-F.; Yu, B. Org. Lett. 2019, 21, 4019.
[27] Yang, W.; Li, B.; Zhang, M.; Wang, S.; Ji, Y.; Dong, S.; Feng, J.; Yuan, S. Chin. Chem. Lett. 2020, 31, 1313.

[28] Luo, K.; Yang, W.-C.; Wei, K.; Liu, Y.; Wang, J.-K.; Wu, L. Org. Lett. 2019, 21, 7851 .

[29] Yu, J.-X.; Niu, S.; Hu, M.; Xiang, J.-N.; Li, J.-H. Chem. Commun. 2019, 55, 6727.

[30] Qin, J.-H.; Yu, J.-X.; Li, J.-H.; An, D.-L. Adv. Synth. Catal. 2019, $361,3974$.

[31] Huang, M.-H.; Hao, W.-J.; Li, G.; Tu, S.-J. Chem. Commun. 2018 $54,10791$.

[32] Gao, Y.; Zhang, P.; Li, G.; Zhao, Y. J. Org. Chem. 2018, 83, 13726.

[33] (a) Xu, J.; Zhang, F.; Zhang, S.; Zhang, L.; Yu, X.; Yan, J.; Song, Q. Org. Lett. 2019, 21, 1112.

(b) Xu, J.; Zhang, S.; Luo, Y.; Zhang, L.; Zhang, F.; Huang, T.; Song, Q. Acta Chim. Sinica 2019, 77, 932 (in Chinese). (许健, 张世焚, 罗荣, 张荔, 张帆, 黄挺菁, 宋秋玲, 化学学报, 2019, 77, 932.)

[34] Wang, L.; Wang, H.; Meng, W.; Xu, X.; Huang, Y. Chin. Chem. Lett. 2020, DOI: 10.1016/j.cclet.2020.02.040.

[35] (a) Liu, X.-C.; Chen, X.-L.; Liu, Y.; Sun, K.; Peng, Y.-Y.; Qu, L.-B.; Yu, B. ChemSusChem 2020, 13, 298.

(b) Jiang, Y.-Q.; Li, J.; Feng, Z.-W.; Xu, G.-Q.; Shi, X.; Ding, Q.-J.; Li, W.; Ma, C.-H.; Yu, B. Adv. Synth. Catal. 2020, 362, 2609.

(Zhao, C.) 